\title{
Enhancement of packet reordering in a mobile stream control transmission protocol for a heterogeneous wireless network vertical handover
}

\begin{abstract}
Future wireless access networks will be heterogeneous wireless network (HWN) environment which consists of various wireless technologies including universal mobile telecommunications system (UMTS) networks and wireless local area networks (WLAN). They are used together through vertical handover (VHO) to ensure global mobility and service continuity. The mobile stream control transmission protocol (mSCTP) layer supports dynamic association reconfiguration. This protocol allows mSCTP endpoints to dynamically add, change and delete IP addresses when the mobile node $(\mathrm{MN})$ is switched between HWNs. During a mSCTP handover, the endpoints of the $\mathrm{mSCTP}$ are required to change the primary link from an old link to a UMTS into a new link to a WLAN. However, due to the disparity between UMTS/WLAN bandwidth, a packet reordering problem will occur when the MN of the mSCTP leaves to a new network. This packet reordering problem can then causes additional drawbacks such as impossibility of growing an mSCTP congestion window, unnecessary fast retransmissions, actual packet losses, and reduced efficiency of the receiving mSCTP. In this paper, we propose a packet reordering model (PRM) that is inserted inside the $\mathrm{MN}$, and works as a special buffer of a large capacity with one input and one output port to receive all transmission sequence numbers (TSNs). It then forwards all incoming data chunks to the MN/WLAN networks after the VHO. The performance of the system is simulated and analyzed using NS-2 simulation tool. The simulation results show that the suggested model enhances the performance throughput and the congestion window of the conventional mSCTP through VHO by handling the packet reordering problem. In other words, the average performance throughout of the proposed PRM scheme is $181.48 \mathrm{Kbps}$ or $16 \%$ increment compared to conventional $\mathrm{mSCTP}$ at $165.54 \mathrm{Kbps}$.
\end{abstract}

Keyword: Vertical handover; 3G; WLAN and UMTS; mSCTP; PRM 